\title{
Focusing Bessel Beams by a Lens with Strong Spherical Aberrations
}

\author{
Vladimir N. Belyi, ${ }^{1}$ Larisa I. Kramoreva, ${ }^{2}$ Muhanna K. Al-Muhanna, ${ }^{3}$ and Nikolai A. Khilo \\ ${ }^{1}$ Laboratory of Optical Diagnostics, B.I. Stepanov Institute of Physics, 68 Nezavisimosti Avenue, 220072 Minsk, Belarus \\ ${ }^{2}$ Department of Medical and Biological Physics, Gomel State Medical University, 5 Lange Street, 246050 Gomel, Belarus \\ ${ }^{3}$ Department of Nanotechnology, King Abdulaziz City for Science and Technology, P.O. Box 6086, Riyadh 11442, Saudi Arabia
}

Correspondence should be addressed to Larisa I. Kramoreva, larisakram@tut.by

Received 24 April 2011; Revised 27 September 2011; Accepted 12 October 2011

Academic Editor: Wonho Jhe

Copyright (C) 2012 Vladimir N. Belyi et al. This is an open access article distributed under the Creative Commons Attribution License, which permits unrestricted use, distribution, and reproduction in any medium, provided the original work is properly cited.

\begin{abstract}
We have carried out the calculation and experimental measurement of the field formed by optical schemes composed of an axicon and lens with strong spherical aberrations. The calculation is performed by the methods of geometrical optics and diffraction integral. A mechanism is revealed, which is responsible for increasing the intensity in the near-axial focus of the doublet. It is shown that the formed bottle beam has a small length and the field at the periphery is of two types: oscillating and smooth ones. The changeover of the field from the bottle beam to a $z$-dependent Bessel beam is traced. The last beam is characterized, in particular, by the Bessel-type structure of its Fourier spectrum.
\end{abstract}

\section{Introduction}

Axicons and their combinations can be used for many applications, and for the first time this came to attention in [1], where a number of specific schemes is considered as well. In particular, a variety of papers are devoted to the calculation and analysis of a focusing scheme consisting of a thin lens and an axicon [2-16]. This combination can be used to obtain a high-quality focal ring. In paper [4] the authors have shown that the irradiance distribution in the transverse plane of the ring is the Gaussian distribution. Rings of such nature seem to be very useful in laser-machining, for example, for the hole drilling application [2]. The axiconlens system is also of interest in the context of feasibility to form an unusual axial distribution of intensities. It is found that, for a certain choice of the Fresnel number of an optical scheme and diameter of the Bessel beam, the axial intensity distribution is characterized by the existence of two distinct foci located to the left and to the right of the geometrical back focal plane $[4,8,10,12,14,16]$.

Between these two foci a region of a very low intensity is located, which is named the bottle beam [17]. During decreasing the distance between the axicon and lens, the far focus disappears and then a hollow beam is formed $[5,7,9$,
$11,12,15]$. Bottle and hollow beams have recently attracted interest because of their applications in optical trapping to trap atoms in the dark region $[18,19]$.

It is interesting to note that there is a region beyond the focal plane where the conical waves again overlap $[8,10]$. Here the initial Bessel-like structure can be reconstructed with a transverse profile expanding while propagation. To create a Bessel beam in this region, another lens is required to rectify the diverging wave front $[3,8]$.

Effects of spherical aberrations in converging and diverging lens-axicon doublets are investigated in paper [6]. Comparisons with aberration-free computations show that higher peak irradiances occur for the converging doublet when spherical aberrations are included. For example, spherical aberrations increase the peak intensity by a factor of 8 for 0.5 deg axicon and $f=100 \mathrm{~mm}$ lens for the of 20 - $\mathrm{mm}$ diameter input beam.

In this work the influence of the spherical aberration is studied for a lens-axicon doublet where the focal length is much smaller $(f \sim 10 \mathrm{~mm})$, and the cone angle of a Bessel beam corresponds to the most frequently used in practice value $(\gamma=1 \mathrm{deg})$. Such a doublet is characterized by a more compact size, which is of importance for possible applications. The theoretical investigation is performed by 


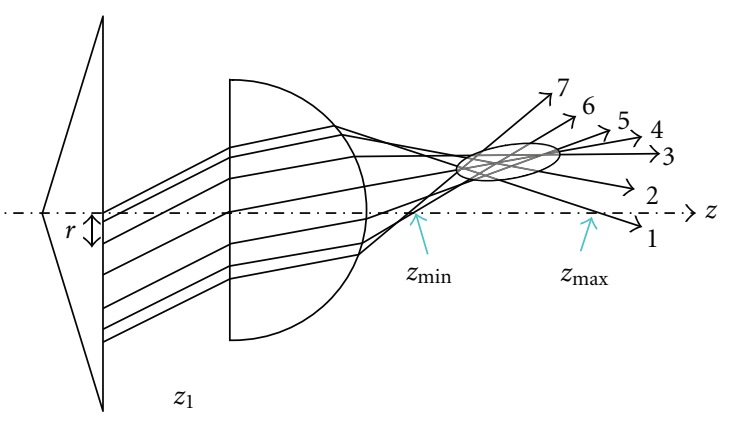

FIGURE 1: Ray diagram for the scheme composed of an axicon and thick lens.

two methods: geometrical optics and diffraction integral. The combination of two methods offers a more clear insight into details of the longitudinal and transverse structure of the field after the lens. Particular emphasis is placed on the description of the transformation of field configurations and, in particular, on the transformation of the bottle beam and $z$-dependent Bessel beam.

\section{Description of the Scheme Using the Ray Tracing Method}

The ray path in the axicon-thick lens scheme is presented in Figure 2. Geometrical constructions can be conveniently performed using the following method. It is suitable to take number 4 as a reference ray, which starts from the center of the half-sphere and, consequently, does not undergo changes in the direction of propagation while emerging from the lens.

In essence, the direction of ray 4 is the analog of the optical $z$-axis in the scheme without any axicon. That is why pairs of rays equally spaced from ray 4 will intersect this ray 4 at the same point. These are the pairs of rays $(3-5 ; 2-6 ; 1-7)$ as exemplified in Figure 1. At that the intersection point of the mentioned pairs of rays will shift toward the lens due to the known spherical aberration effect, which is just presented in Figure 1. In the scheme the spherical aberration will lead to the spreading of the focal ring in comparison with the case where a thin lens is used. One of two ellipse-shaped sections of this ring by the figure plane is presented in Figure 1.

The annular field may be regarded as an optical source generating a divergent light beam. The direction of ray 3 in this beam is characterized by the fact that the energy propagates behind the lens in parallel to the $z$-axis. All rays between 1 and 3 are seen to intersect $z$-axis to form a conical beam of rays in the space. The cone angle therewith decreases with increasing $z$. Ray 1 is the last one as it issues out of the center of the axicon. It should be noted that depending on the relationship between the cone angle of axicon, distance $z_{1}$, and diameter of lens $2 R$, this ray may undergo the total internal reflection and then the marginal ray will issue out of the point located outside the optical $z$-axis. This ray 1 determines the far boundary $z_{\max }$ of the dark area in the

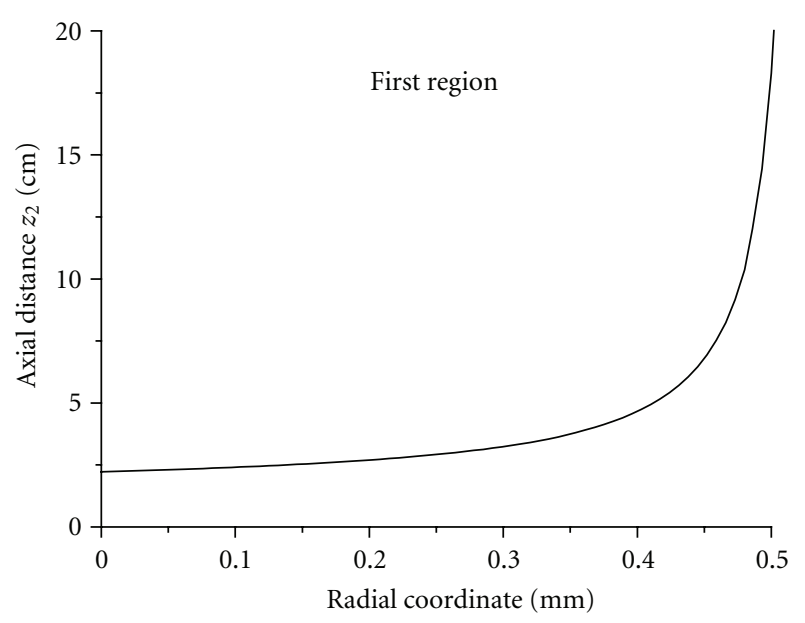

(a)

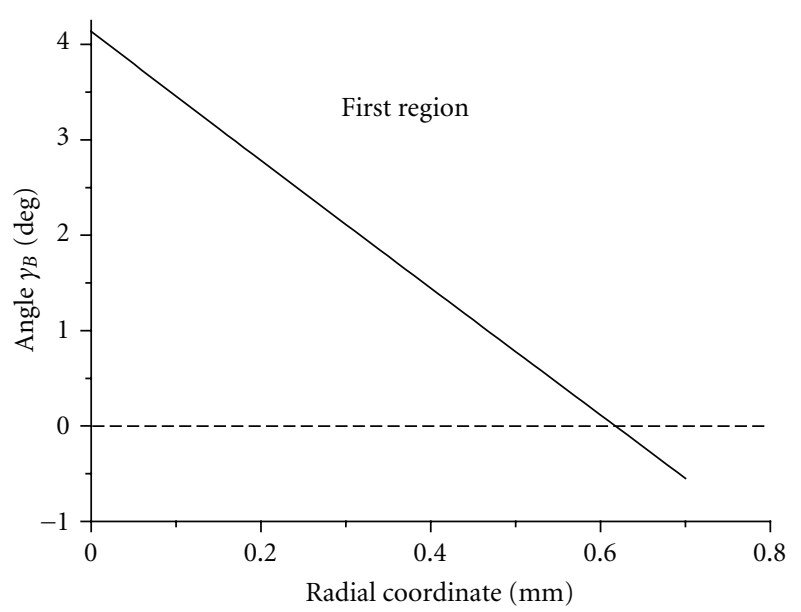

(b)

Figure 2: Two parameters of the output light ray from area I, that is, the distance $z_{2}$, at which it intersects the optical axis (a) and inclination angle $\gamma_{B}$ (b) depending on the radial coordinate $r$ of the ray in the axicon plane.

vicinity of the $z$-axis. Its near boundary is determined by ray 6 . It is characterized by the fact that all rays being lower than ray 6 intersect the optical axis closer to the lens, that is, at a smaller distance $z$. The presence of this optical effect follows from the mathematical calculation of the ray path in the scheme. As a result of the calculation, we have obtained two parameters of the output ray, namely, (i) the distance $z_{2}$ between the lens and point of intersecting of the optical axis by the ray and (ii) the angle $\gamma_{B}$ of crossing of the $z$-axis by the ray. Final equations for $z_{2}$ and $\gamma_{B}$ slightly differ for three types of rays.

(I) The ray enters the lens and emerges from it above the optical $z$-axis (see Figure 1).

(II) The ray enters the lens and emerges from it below the optical $z$-axis.

(III) The ray enters below the optical $z$-axis and leaves above this axis. 
In case I we have

$$
\begin{gathered}
z_{2}=\frac{h_{2}}{\operatorname{tg}\left(\gamma_{B}\right)}+L-R, \\
\gamma_{B}=\left(\gamma_{\text {out }}-\gamma_{0}\right) .
\end{gathered}
$$

Here $h_{2}=\cos ^{2}\left(\gamma_{2}\right)\left(c_{1} h_{1}+c_{2} \sqrt{h_{1}^{2}-\left[h_{1}^{2}-R^{2} \operatorname{tg}^{2}\left(\gamma_{2}\right)\right] / \cos ^{2}\left(\gamma_{2}\right)}\right)$ is the radial coordinate of the ray emerging from the lens, $h_{1}=z_{1} \operatorname{tg}\left(\gamma_{1}\right)-r$ is the radial coordinate of the ray at the input of the lens, and $r$ is the radial coordinate of the ray in the plane of the axicon; the incidence and refraction angles are equal to $\gamma_{1,2}$ where $\sin \left(\gamma_{1}\right)=n \sin \left(\gamma_{2}\right) ; n$ is the refraction index; $\gamma_{\text {in }}=\left(\gamma_{0}-\gamma_{2}\right)$ is the incidence angle between the ray and spherical surface; $\gamma_{\text {out }}=\sin ^{-1}\left(n \sin \left(\gamma_{\text {in }}\right)\right)$ is the angle of the output ray with the sphere surface; $\gamma_{0}=\operatorname{tg}^{-1}\left(h_{2} / L\right)$, $L=\sqrt{R^{2}-h_{2}^{2}}, c_{1,2}=1$. For case II the presented formulas are modified in accordance with the substitutions: $c_{1}=1, c_{2}$ $=-1, h_{1}=r-z_{1} \operatorname{tg}\left(\gamma_{1}\right), \gamma_{\text {in }}=\left(\gamma_{0}+\gamma_{2}\right)$. In case III the substitutions are as follows: $c_{1}=-1, c_{2}=1, h_{1}=r-z_{1} \operatorname{tg}\left(\gamma_{1}\right)$, $\gamma_{\text {in }}=\left(\gamma_{2}-\gamma_{0}\right), \gamma_{B}=\left(\gamma_{\text {out }}+\gamma_{0}\right)$.

Note that light rays belonging to the area I always intersect the optical axis, and rays from area III never intersect the axis. The angle $\gamma_{B}$ for them in the presented formulas will be positive. The rays from area II may or may not intersect the axis $z$. Here if there is the intersection of the axis, the angle $\gamma_{B}$ is positive, otherwise it is negative.

Let us perform the numerical calculation for a specific case, which corresponds to the experiment. Let $R=7.5 \mathrm{~mm}$, $\gamma_{1}=1 \mathrm{deg}, z_{1}=4 \mathrm{~cm}$. At $n=1.868$ we receive $\gamma_{2} \approx 0.53 \mathrm{deg}$. For small cone angles the formula $h_{2} \approx c_{1} h_{1}+c_{2} \operatorname{Rtg}\left(\gamma_{2}\right)$ is valid with a high degree of accuracy. Let us calculate the parameters of beams typical for this case. Ray 1 (see Figure 1) issues out of the axicon center and determines the lower boundary (in values of the radius $r$ ) of area I. Its parameters at the output (see (1) and (2)) in this case are $z_{2} \approx 10.6 \mathrm{~mm}$, $\gamma_{B} \approx 2$ deg. Consequently, the far boundary of the dark area is $z_{\max } \approx 10.6 \mathrm{~mm}$. Ray 3 is also situated in area I. Its upper boundary $r_{1 \max }$ is found from the equation $h_{1}\left(r_{1 \max }\right)=0$ and corresponds to ray 4 in Figure 1. From the above formula we find $r_{1 \max }=z_{1} \operatorname{tg}\left(\gamma_{1}\right)$ and the numerical value $r_{1 \max } \approx$ $0.7 \mathrm{~mm}$. The plots in Figure 2 show the distance $z_{2}(r)$ and angle $\gamma_{B}(r)$ for area $\mathrm{I}$ in relation to the radial coordinate $r$.

Figure 2 shows that the beam issuing the center of the axicon on passing through the optical scheme intersects the optical axis under a maximal angle of approximately 4 degrees. This ray, as is mentioned above, determines the far boundary of the dark area. As the radius $r$ increases, the inclination angle of output rays decreases and the distance where they intersect the axis becomes larger. At $r=0.62 \mathrm{~mm}$ the angle $\gamma_{B}$ is equal to zero and the distance is $z_{2} \rightarrow \propto$. These parameters determine ray 3 in Figure 1. On further increasing $r$, the output beams have the off-axis direction. Note that beam 3 is placed at the distance $h_{23}=0.15 \mathrm{~mm}$ from the optical axis and its intersection with ray 4 analogues to the optical axis (see above) is achieved at the distance $z_{34}=h_{23} \operatorname{tg}^{-1}\left(\gamma_{2}\right)-R$ which is equal to $8.9 \mathrm{~mm}$.

It should be noted that this distance is equal to the paraxial focus. Figure 1 shows that the coordinates $\left(h_{23}, z_{34}\right)$ determine approximately the position of the annular field behind the lens. As is seen, the diameter of the dark area is very small. It is also worth noticing that when comparing Figures 2(a) and 2(b), it follows that in area I the angle $\gamma_{B}$ decreases with increasing the distance $z_{2}$, or the derivative $d \gamma_{2} / d z_{2}<0$. Such a behavior of light beams will be characterized by the term "negative angular dispersion."

Now let us consider area II in more detail. Its lower boundary in value of the radial coordinate $r$ is determined by the formula $r_{2 \min }=z_{1} \operatorname{tg}\left(\gamma_{1}\right)+\operatorname{Rtg}\left(\gamma_{2}\right)$. This formula has been obtained using $h_{2}(r)=0$ in the small angle $\gamma_{1}$ approximation. The calculation according to this equation gives $r_{2 \min } \approx 0.77 \mathrm{~mm}$. The upper boundary $r_{2 \max }$ here is determined either by the radius of a beam incident on the axicon or by the effect of the total internal reflection. The second limitation provides the equation for determining the radius $\gamma_{\text {out }}(r)=\pi / 2$ from which we find $r_{2 \max } \approx 5.25 \mathrm{~mm}$. This value is lower than the radius of the input beam. Therefore in the particular case under study the limitation of area II occurs due to the total internal reflection on the spherical surface.

In Figure 3 the angular and spatial coordinate of the rays from area II at the point of crossing by them of the optical $z$-axis are plotted.

As is seen in Figure 3(a) the spatial coordinate is nonmonotonously dependent on $r$. For paraxial beams (small values of $r$ ) the angle dispersion of rays is positive, that is, the angle $\gamma_{B}$ increases when the increasing $z_{2}$ becomes larger. The maximum value of $z_{2}$ is equal approximately to $7.3 \mathrm{~mm}$ and is realized at $r=2 \mathrm{~mm}$. On further increasing $r$ the negative angle dispersion of rays takes place up to the critical angle of the total internal reflection. The curve in Figure 3(a) shows a rather wide flat area of about $2 \mathrm{~mm}$ in length. Here the angle dispersion is large and the derivative $d z_{2} / d r$ is small, which is indicative of a high concentration along the optical axis of rays with various values of the angle $\gamma_{B}$. This has to lead to an increase in the axial intensity of radiation in the mentioned area. On the whole two-valuedness of the function $z_{2}(r)$ means that each point of the optical axis here is intersected by two cones of rays. At the point of the maximum of the curve $z_{2}(r)$ two cones merge into one cone and ray 6 passes through this point in Figure 1. This allows one to estimate

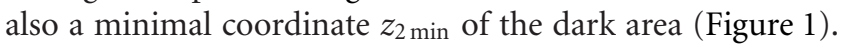
The calculation for the presented above parameters of the scheme gives $z_{2 \mathrm{~min}}=7.3 \mathrm{~mm}$. Thus, the bottle beam formed behind the lens is localized within an interval of $(7.3 \div 10.6)$ mm.

\section{Field Calculation with Application of the Diffractional Integral}

As it is seen from the previous section, the method for ray optics allows one to obtain a number of simple and visual results, concerning the structure of the field, formed by the axicon-thick lens system. If there is a necessity of studying the field structure in more detail, diffractional integrals should be used. For all, in analogy to the previously given facts, the basic field characteristics could be withdrawn from 


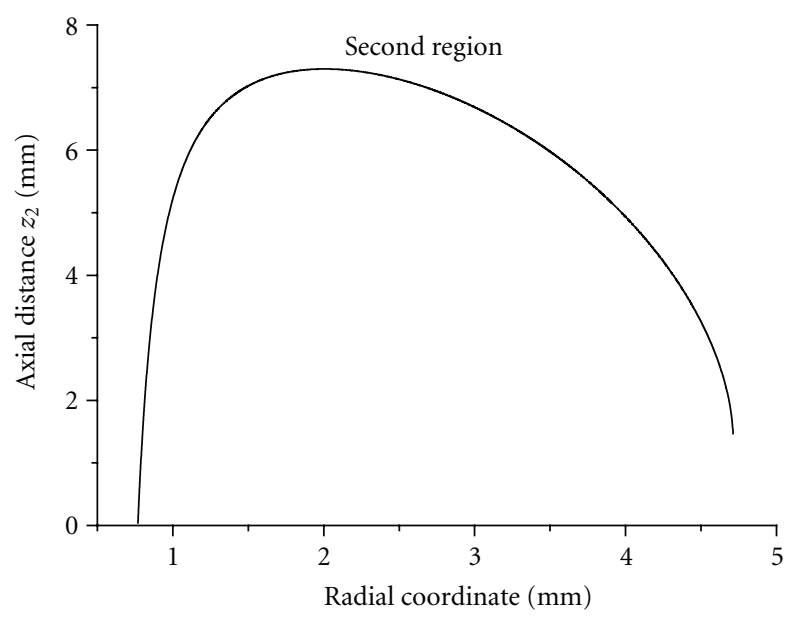

(a)

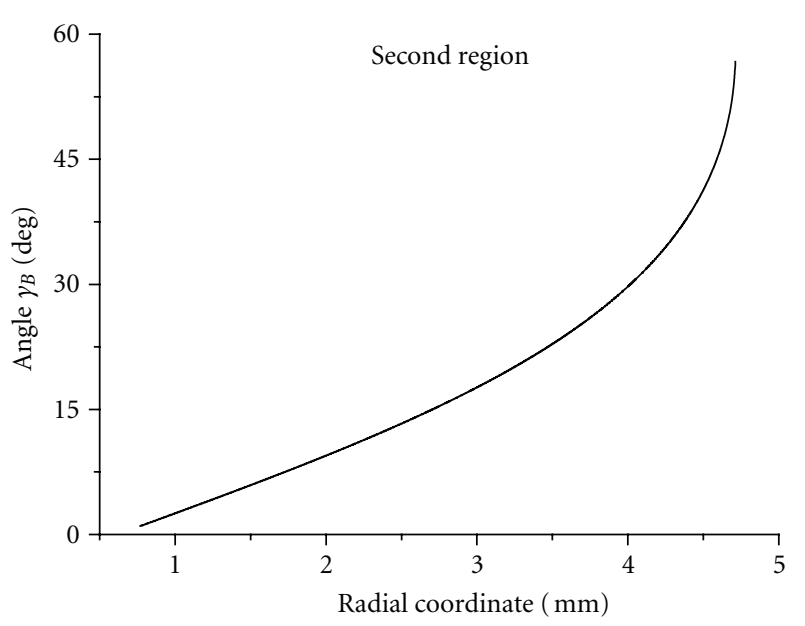

(b)

FIGURE 3: Parameters of the light ray belongs area II, that is, the distance $z_{2}$ at which it intersects the optical axis (a) and inclination angle $\gamma_{B}$ (b) versus the radial coordinate of the ray in the plane of axicon.

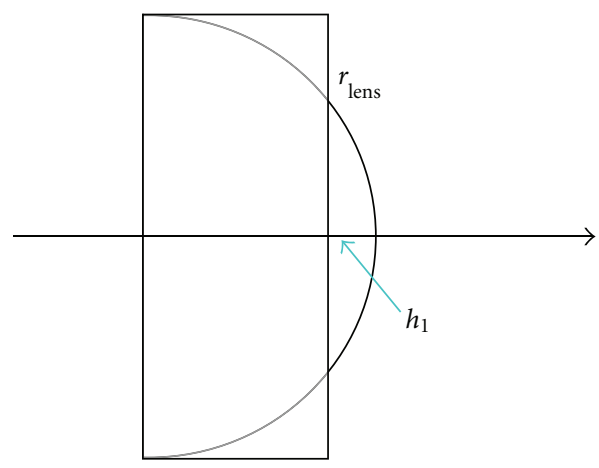

FIgURE 4: To the definition of the thick lens effective aperture.

the analysis of the diffractional integral in the scalar form. The advantage of this approach in comparison with the application of vector diffraction theory consists in simplicity and visuality of the obtained results. At this, however, a preliminary analysis of the borders of application of scalar diffraction theory for the description of the scheme in Figure 1 is necessary. As it is known, paraxial optics approximation has an accuracy not high enough, when the radiation is propagated under the large angle to the optical axis. Then it follows from Figures 2 and 3 that $z$-range where the paraxial approximation is valid is large enough, namely, angle $\gamma_{B}$ transcends the paraxial approximation only near the lens, that is, at $z \leq 1 \mathrm{~cm}$.

The second approximation concerns the kind of the function of lens transmission. In the given paper it will be expressed as follows: $\tau_{L}(r)=t(r) \exp \left[k_{0}(n-1) \sqrt{R^{2}-r^{2}}\right]$, where $t(r)$ is Fresnel transmission coefficient on the interface spherical surface-air. The phase function, included in this equation, considers spherical aberrations of all orders. An effective aperture radius $r_{\text {lens }}$ can be calculated from the geometrical optics equations (1) and (2). From the definition of angle $\gamma_{0}$ it follows, that for the marginal beam $\sin \left(\gamma_{0}\right)=$ $r_{\text {lens }} / R$. Taking into consideration the fact that radii $\eta_{\text {lens }}$ and
$R$ are of the same order (see Figure 4), we have the inequality $\gamma_{2} \ll \gamma_{0}$. Then the condition of total internal reflection will have the form $\sin \left(\gamma_{0}\right) \approx 1 / n$, from which the equation $r_{\text {lens }} \approx$ $R / n$ follows. In this particular case we have $r_{\text {lens }} \approx 4 \mathrm{~mm}$. At that, the thickness of lens is $h_{\text {lens }} \approx 1.2 \mathrm{~mm}$. As we can see, the effective lens is thin enough and its description with phase transmittance function is reasonable. The remaining part of the thick lens can be changed with usual plane-parallel plate (see Figure 4). As a result the diffraction equation, considering light propagation from input of the axicon up to input of the effective lens, will be the following:

$$
\begin{aligned}
a(r)=\frac{i k_{0}}{z_{1}+L / n} & \int a_{0}\left(r_{1}\right) \exp \left(-i k_{0} \gamma r_{1}+i k_{0} \frac{r^{2}+r_{1}^{2}}{2\left(z_{1}+L / n\right)}\right) \\
& \times J_{0}\left(\frac{k_{0} r r_{1}}{z_{1}+L / n}\right) r_{1} d r_{1},
\end{aligned}
$$

where $a_{0}(r)$ is amplitude of the field, incident on the axicon, $J_{0}(x)$ is Bessel function of the first type of zero order.

To calculate the field behind the lens, amplitude (3) has been multiplied by the given above function of the lens transmission, and further it has been calculated with the diffractional integral in free space.

3.1. Intensity Distribution along the Optical Axis. The result of calculation the intensity distribution along the optical axis is given in Figure 5. As it follows from the comparison of numerical values of intensity in maxima in the Figures 5(a) and 5(b), near the point $z \approx 12.86 \mathrm{~mm}$ a strong light energy concentration takes place. When moving away from the given point towards the lens the intensity decreases sharply to the value close to zero.

At the same time when approaching the lens the intensity decreases slower. As the result, the longitudinal axial maximum size is $\sim 0.2 \mathrm{~mm}$. Intensity has not been calculated on closer distance to the lens owing to the supposed loss of the calculation accuracy in nonparaxial region. The maximum 


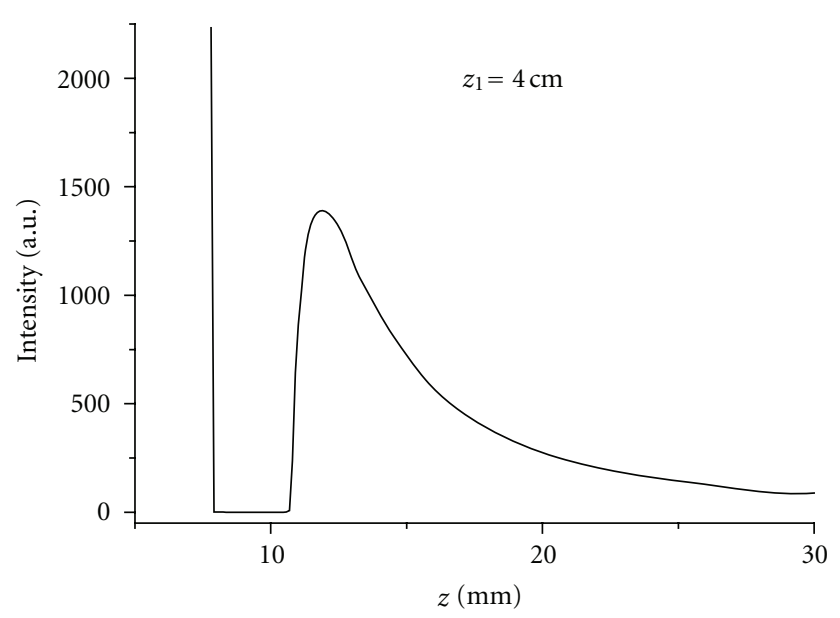

(a)

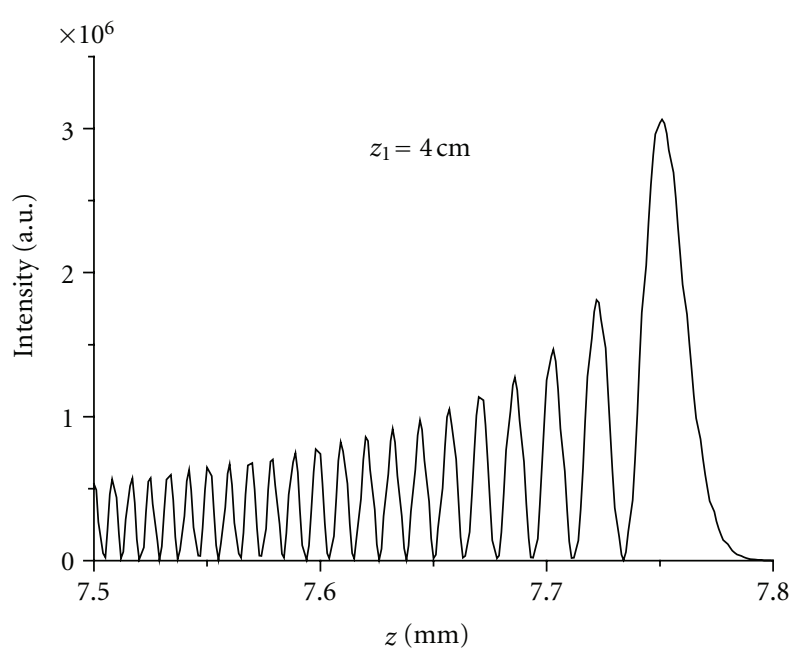

(b)

FIGURE 5: Dependence on-axis light intensity behind the thick lens in the scheme in Figure 1 when the distance between the axicon and the lens is $4 \mathrm{~cm}$.

described above obviously corresponds to the described in the Section 1 case, when the derivative $d z_{2} / d r$ is equal or close to zero (see Figure 3(a)).

When moving away from the lens, as it is seen from the Figure 5(a), a dark area is clearly seen. Its near and far borders are equal to $z_{\min } \approx 7.78 \mathrm{~mm}, z_{\max } \approx 10.6 \mathrm{~mm}$, and it corresponds well to the given above estimation, made on the basis of ray optics. The dark area ends with wider and less intensive maximum (Figure 5(a)), after which the intensity decreases steadily with the increase of the distance $z$.

3.2. Transverse Intensity Distribution along the Optical Axis. Transverse intensity distribution on various distances from the lens has been also calculated. The aim was to reveal tendencies in intensity behavior, and, in particular, to reveal here the opportunities of Bessel-type beams formation. Generally, the transversal structure of the field has been classified by us on four types. The first field type is given in Figure 6. It is realized near the basic maximum of the axial intensity, shown in Figure 5(b).

Distance $z$ for the curve in Figure 6(a) strictly corresponds to the given above intensity maximum. As we can see, here there is an ordered ring structure with little number of rings. The reason for a small transverse size of the beam (radius is $\sim 10 \mu \mathrm{m}$ ) can be understood from the fact that in the given area the interference of large number of conical beams with different cone angles takes place (see Figure 3 and its discussion in Section 1). At that near the axis the constructive interference prevails, which leads to the formation of intensive maximum. The plot in Figure 6(b) has been drawn for the point of the first interference minimum, shown in Figure 5(b). Here the field structure is more random and it expresses some contrast to the ordered structure in the previous figure. The plots in Figures 6(c) and 6(d) illustrate transition to the bottle beam. They are characterized by the destructive interference domination in the near-axis region and the tendency for formation of ordered multi-ring field in the periphery.

Figure 7 (a) presents an example of the developed peripheral field in the initial area of bottle beam existence. Regular multi-ring structure of the intensity distribution with modulation depth equaling to 1 points out, probably, the existence of the analytical description of such a structure. With the growth of $z$ the width of the dark area grows (compare with Figure 1), but generally the deformation of ordered structure starts and this structure has the tendency to become a single-ring field. The intermediate state of the field is shown in Figure 7(b). Here, the width of bottle beam is large (about $150 \mu \mathrm{m}$ ), the intensity modulation depth decreases, and nonoscillating component appears.

Figure 8 shows the developed single-ring field being a periphery of bottle beam. With the growth of $z$ and approaching the far border of bottle beam single-ring field is compressed to the optical axis, forming the area with very strong intensity radial gradient (Figure 8(b)). The absence of oscillation specifies the fact that given field has a conical nature with narrow angular spectrum. Such a field is typical for a Bessel beam focusing with the thin lens [12]. It is proved in Figure 2, where it is shown that in given distances the conical field is a paraxial one, and the cone angle decreases with the growth of $z$.

During the process of compression, single-ring field reaches the optical axis, two conical beams appear as a result: the first one converging to the axis, the second one diverging from it. At this a constructive interference and the intensity maximum formation take place directly on the axis. The first stage of overlapping of the stated two beams is given in Figure 9(a). Generally, only axial interference maximum is formed here. With the growth of $z$ the area of overlap, accordingly, grows, and this leads to the increase of Besseltype beam diameter (Figures 9(b), 9(c) and 9(d)). However, owing to the decrease of a cone angle of two conical beams with the growth of $z$, the cone angle of a Bessel-type beam 


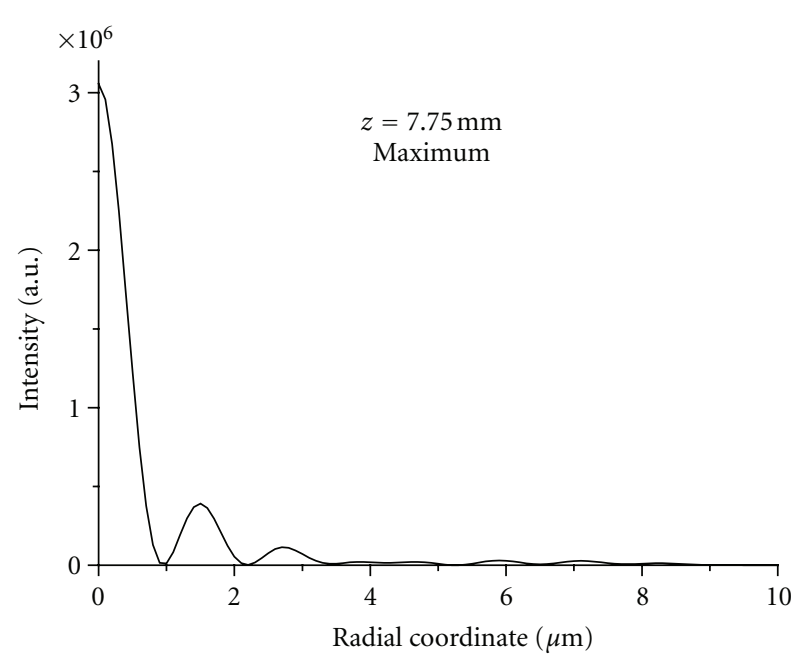

(a)

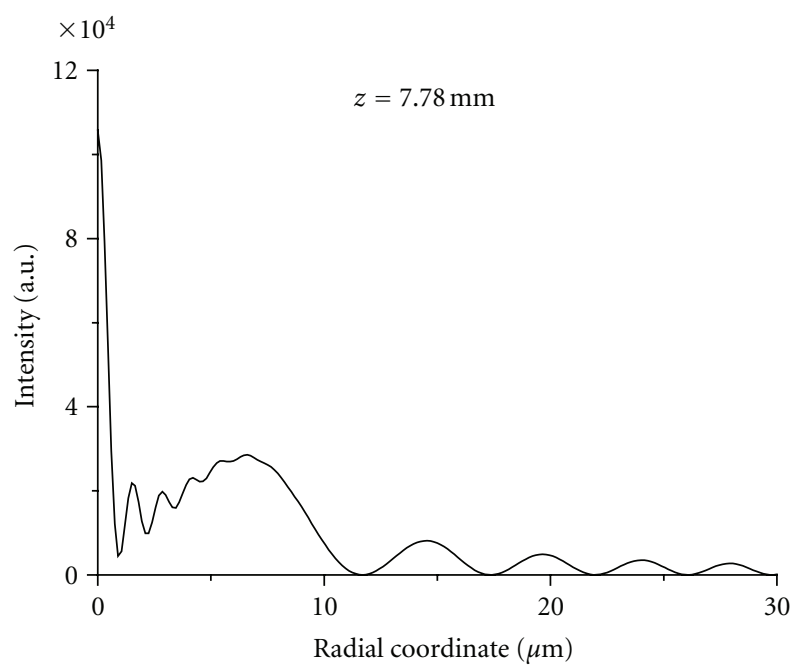

(c)

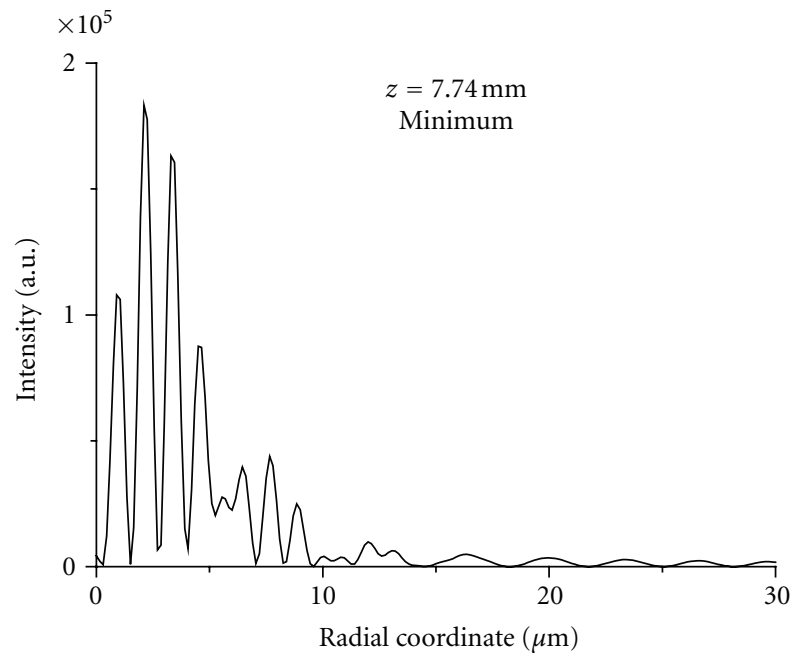

(b)

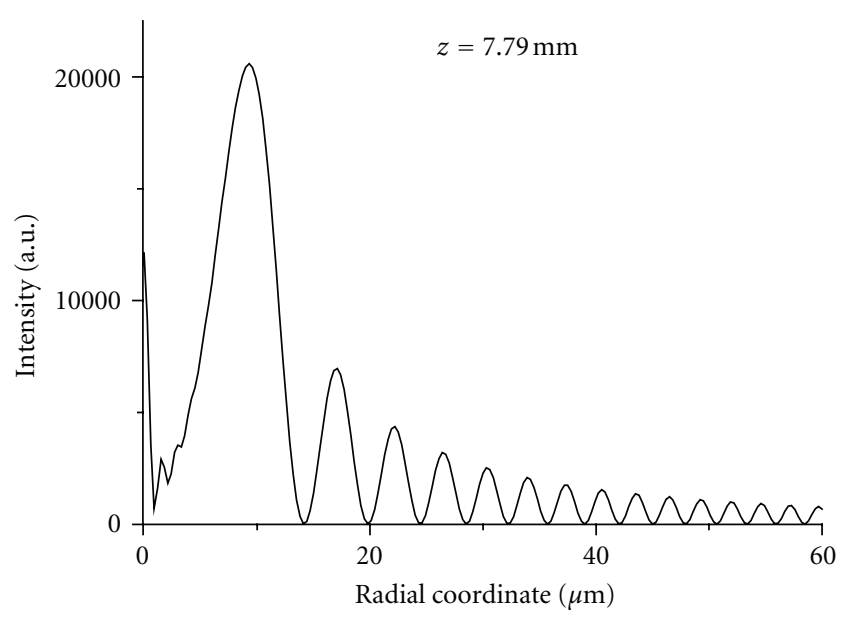

(d)

FIGURE 6: Transverse-oscillating light field with axial intensity maximum.

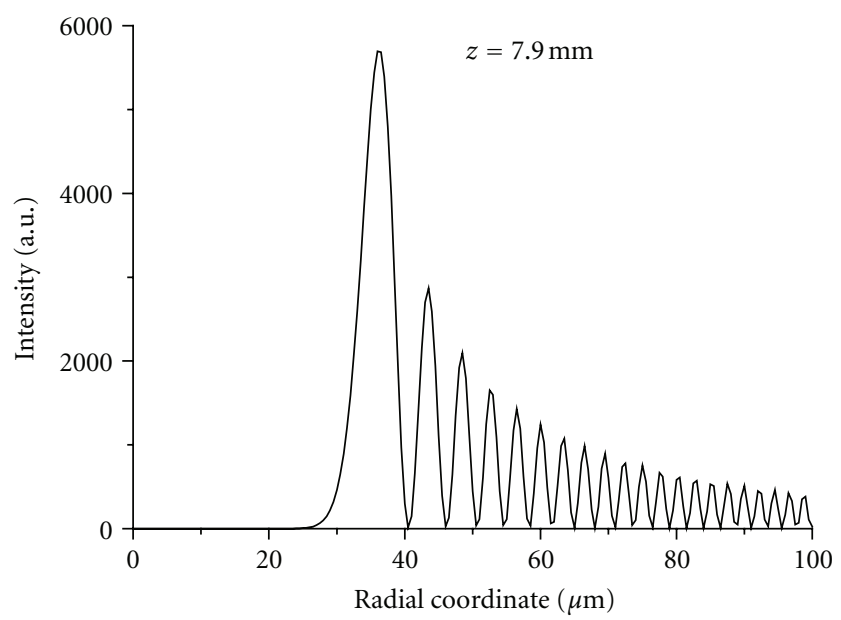

(a)

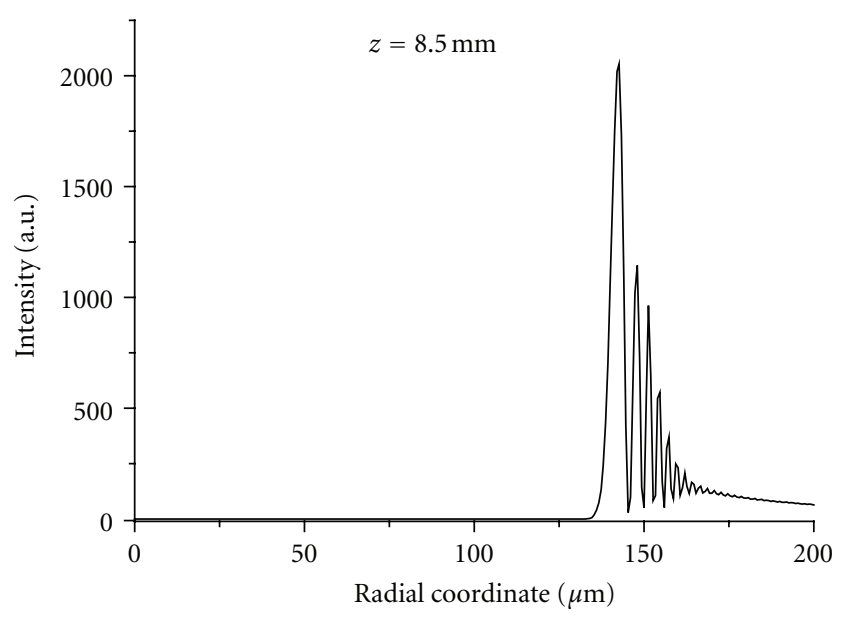

(b)

Figure 7: Multiring light field in the region of optical bottle with wide axial intensity minimum. 


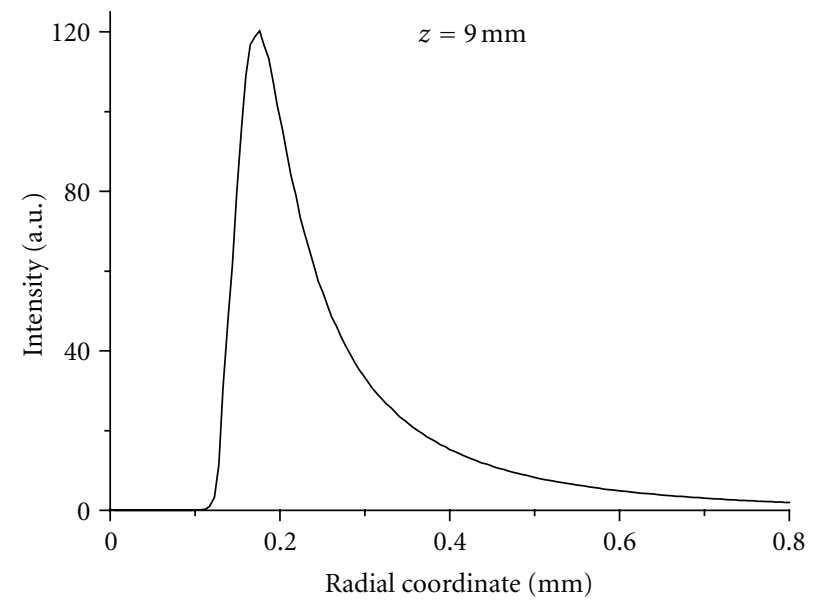

(a)

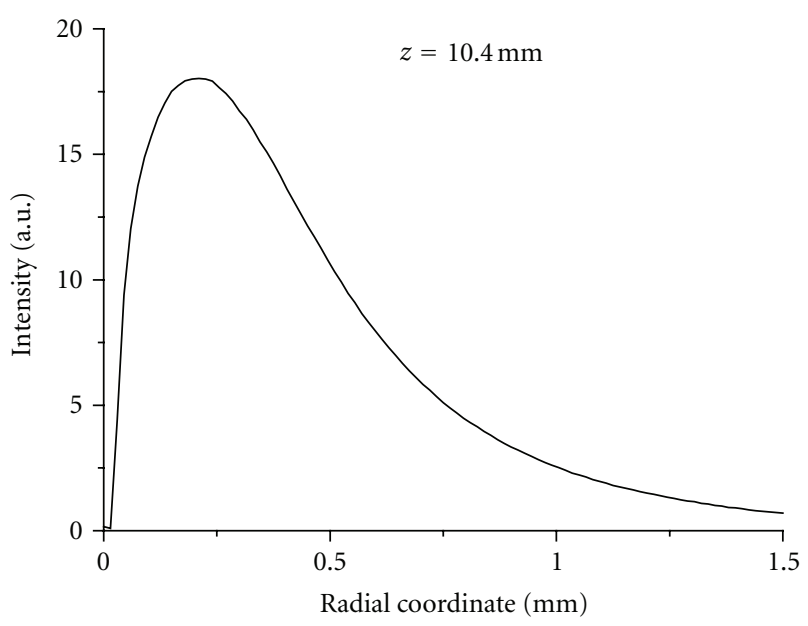

(b)

FIGURE 8: Single-ring light field in the region of optical bottle with narrow axial intensity minimum.

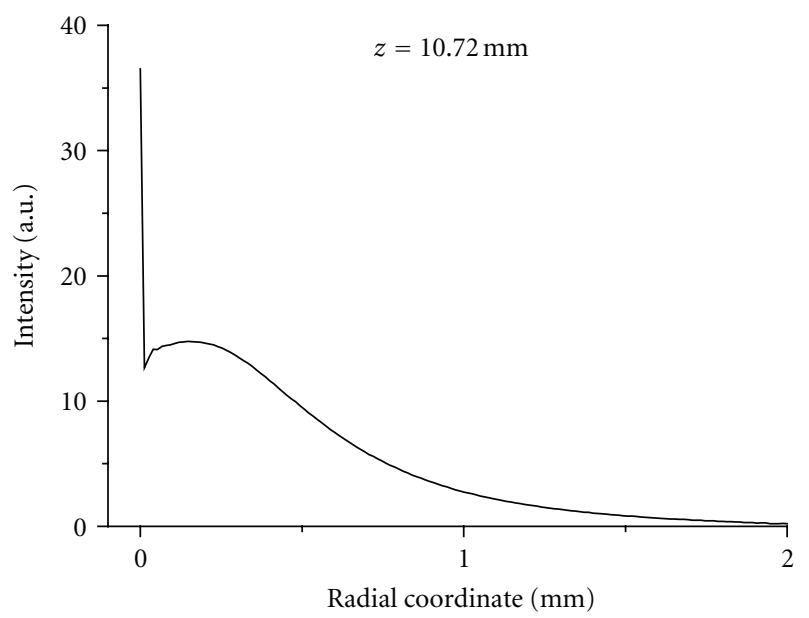

(a)

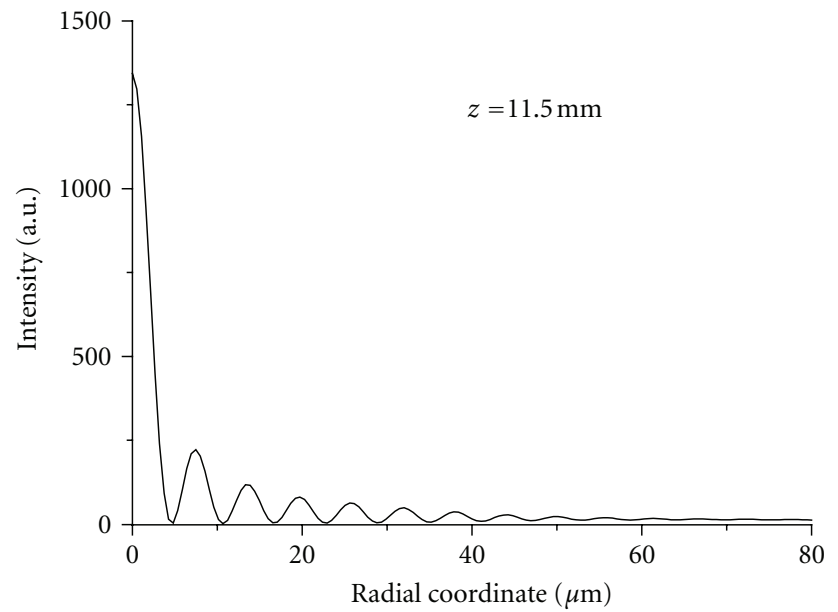

(c)

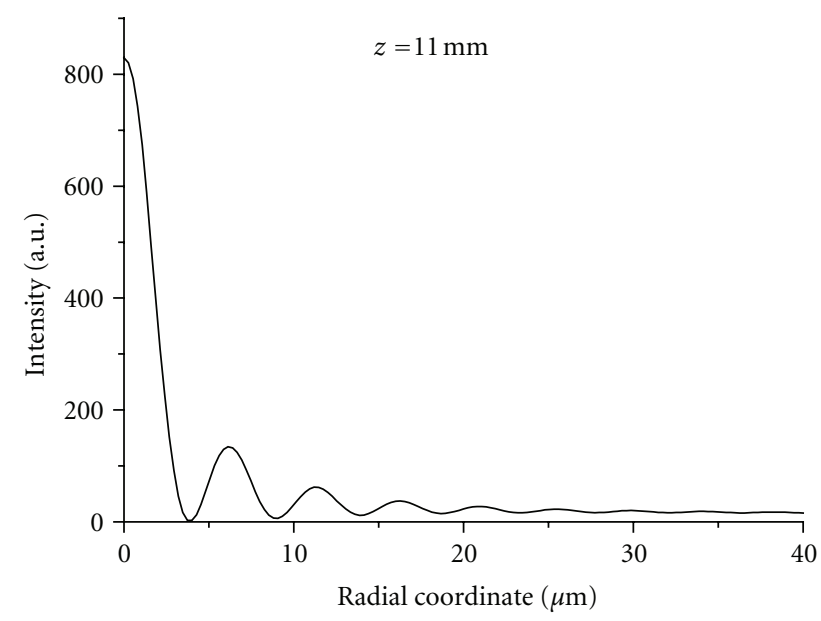

(b)

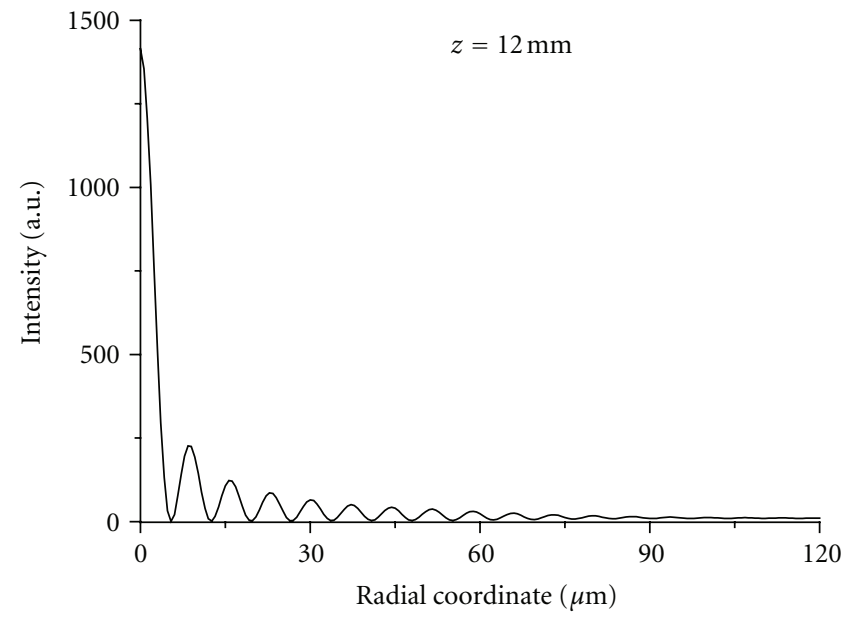

(d)

FIGURE 9: The area of formation and existence of Bessel light beam with the cone angle, depending on a distance. 


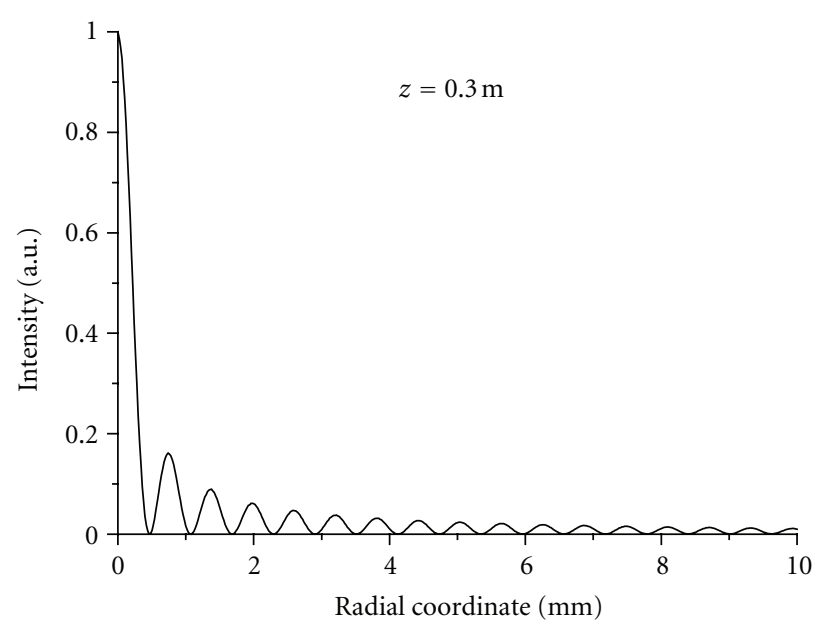

(a)

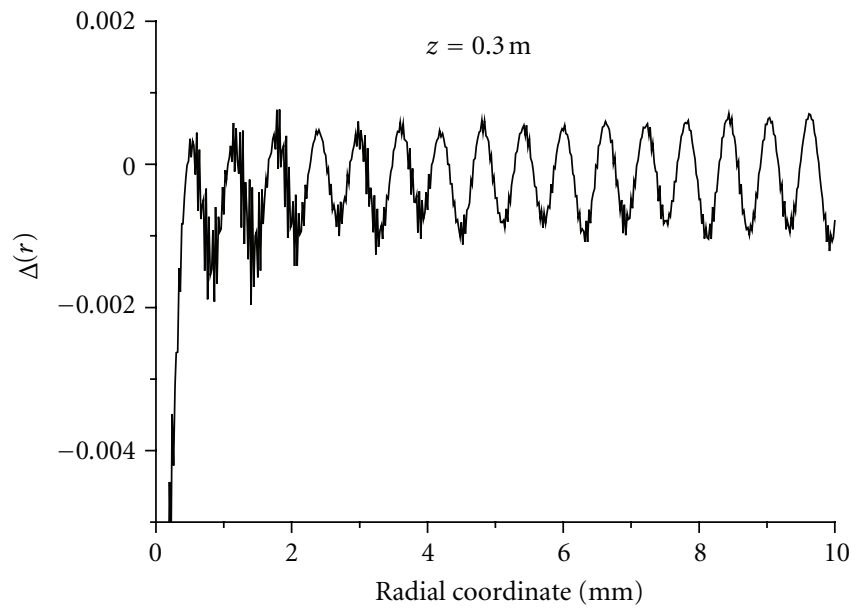

(b)

FIGURE 10: Intensity distribution of a quasi-Bessel beam, formed by the axicon-thick lens system at a distance of $0.3 \mathrm{~m}$ (a). Difference of the intensities of quasi-Bessel beam and a standard one (b).

decreases, and ring diameters grow according to the distance. This fact leads to a nonlinear dependence between the total number of beam rings and its diameter.

3.3. Calculation of the Far Field. The similarity of the transverse structure of the formed field to the Bessel beam is of great importance. According to the calculations given above, with the distance growth it is possible to expect the formed field approaching zero-order Bessel beam. In Figure 10(a) an example of a normalized intensity distribution $I(r)$ at a distance of $1 \mathrm{~m}$ from the lens is shown. The comparison of function $I(r)$ with the standard Bessel functions of the kind $J_{0}^{2}\left(k_{0} \gamma_{B} r\right)$ has been conducted. Angle $\gamma_{B}$ is calculated with formula (2). For example, at $z=0.3 \mathrm{~m}$ it equals to $5.2 \cdot 10^{-4}$, $\mathrm{rad}$, and at $z=20.5 \mathrm{~m}$ the angle $\gamma_{B}$ equals to $3.1 \cdot 10^{-4} \mathrm{rad}$. In Figure 10(b) the differential function $\Delta(r)=I(r)-J_{0}^{2}\left(k_{0} \gamma_{B} r\right)$ for the case $z=0.3 \mathrm{~m}$ is shown. As we can see, the value of the given function does not exceed the value $1 \%$, and this fact indicates a high degree of intensity distribution similarity to Bessel one. With the growth of $z$ the approximation accuracy becomes stronger.

Except the investigation of intensity function $I(r)$, the spatial spectrum of the field behind the thick lens has been found. A standard scheme of Fourier lens transformation with long-focused lens has been calculated for this. The obtained Fourier-spectrum intensity distribution in analogy with the given in Figure 10 one is described by Bessel function with high accuracy. It shows particularly the fact that in a distance $z=0.3 \mathrm{~m}$ a far field of radiation generated by the system axicon-thick lens is realized. However, a much more important result consists in the fact that the given scheme forms a so-called $z$-dependent Bessel beam in far field [20]. Such a beam is characterized by its local transverse intensity distribution being Bessel one, but the cone angle of the beam decreases monotonously inversely to the distance $z$. Fourier-spectrum of $z$-dependent Bessel beam is not a ring one, as it is for a standard Bessel beam, but Bessel one

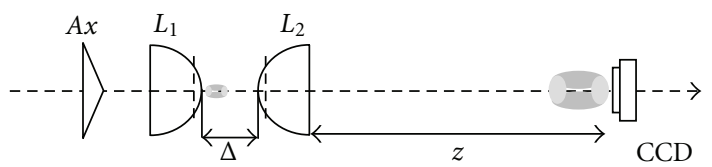

FIGURE 11: Experimental setup for production of quasi-Bessel light beam: $A x$-axicon; $L_{1,2}$-half-ball lenses.

as well. Such a spectrum behavior makes the $z$-dependent Bessel beam similar to the beams of Gaussian type.

\section{Experimental Setup and Results}

The scheme of experimental setup is shown in Figure 11. A Gaussian beam from a He-Ne laser $(\lambda=0.63 \mu \mathrm{m})$ is expanded by the collimator 2 up to diameter of $2 \mathrm{~cm}$. Linear axicon $A x$ has the apex angle $\alpha=186 \mathrm{deg}$. The thick planeconvex lens $L_{1}$, having the form of half-ball with curvature radius $7.5 \mathrm{~mm}$ and refractive index $n \approx 1.87$, is placed behind the axicon in the area of the existence of conical and Bessel beams. To register the fine structure of the field that is localized in proximity to the lens, use was made of a simple scheme of magnification with the help of a short-focus lens 2 that is identical to lens 1 . In the process of measuring, lens 2 moved along the optical axis whereas the CCD-matrix occupied a fixed position. The distance between the axicon and lens was also unchanged and equal to $4 \mathrm{~cm}$.

The maximal displacement of lens 2 in the measuring process amounted to $2 \mathrm{~cm}$, and the average distance $z$ was equal to $121 \mathrm{~cm}$. Because of a great value of $z$, the image formation was brought about by means of the near-axial region of lens 2; therefore, aberration-induced distortions were negligible. The average magnification was about 130 and changed insignificantly at small shifts of lens 2 .

The basic results of measurements are shown in Figures 12 and 13. Figures 12(a1)-12(a3), 12(b1)-12(b3) illustrate the situation appropriate to Figure 3(a) where there takes 

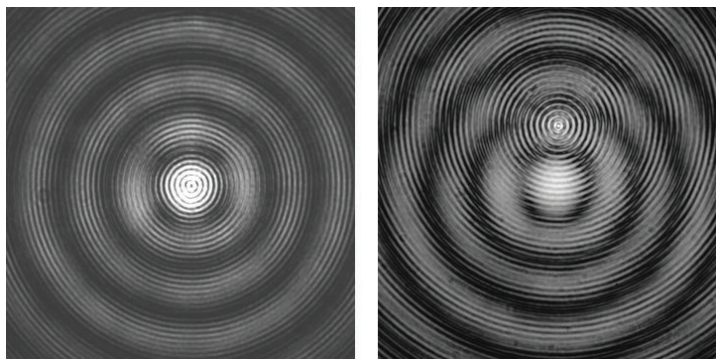

$z=3.3 \mathrm{~mm}$

(a1)

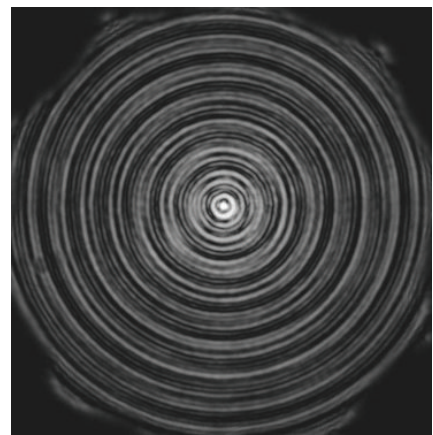

$z=4.3 \mathrm{~mm}$

(a2)

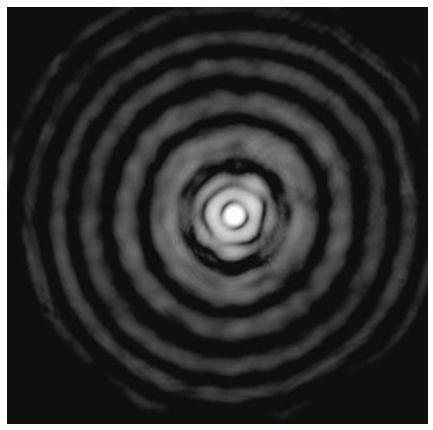

$z=7.3 \mathrm{~mm}$

(a3)

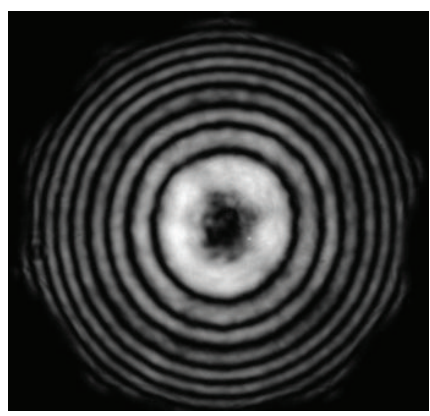

$z=7.8 \mathrm{~mm}$

(a4)

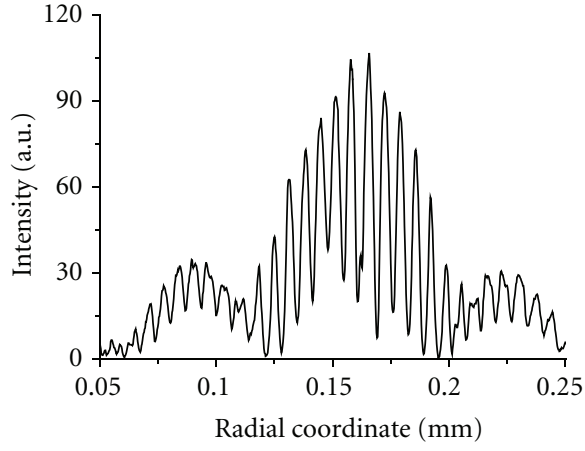

(b1)

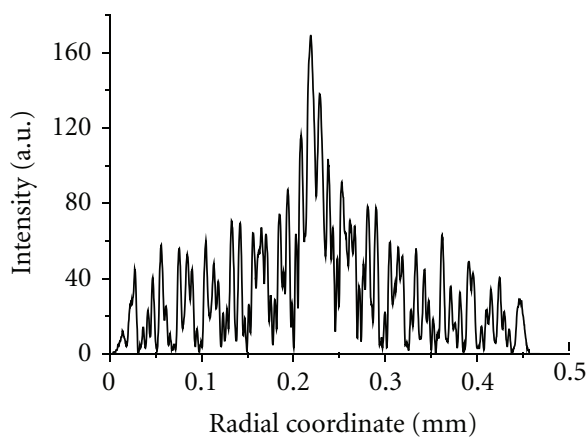

$z=4.3 \mathrm{~mm}$

(b2)

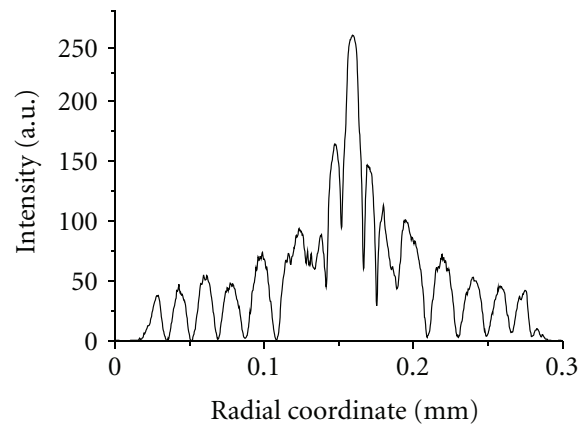

$z=7.3 \mathrm{~mm}$

(b3)

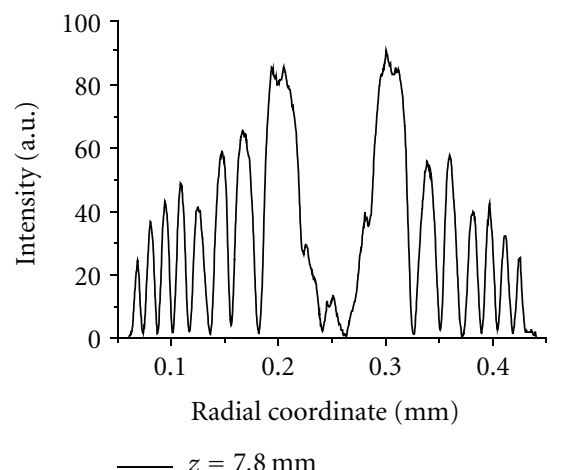

(b4)

(a)

FIgURE 12: Continued. 


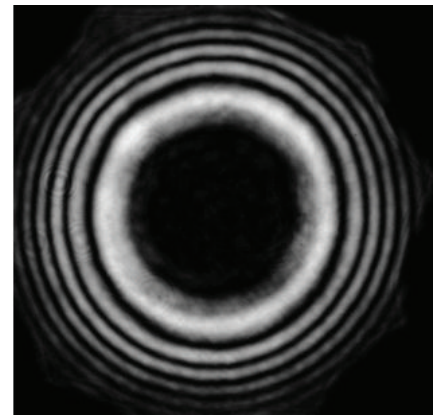

$z=8.3 \mathrm{~mm}$

(a5)

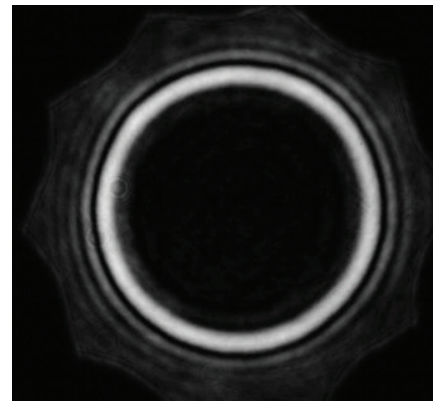

$z=8.8 \mathrm{~mm}$

(a6)

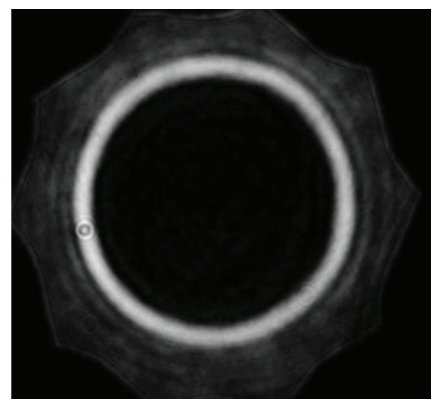

$z=9.3 \mathrm{~mm}$

(a7)

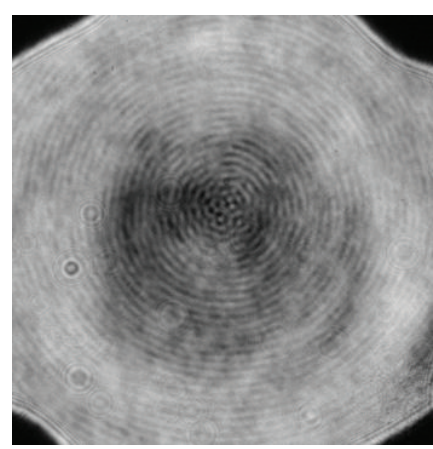

$z=10.3 \mathrm{~mm}$

(a8)

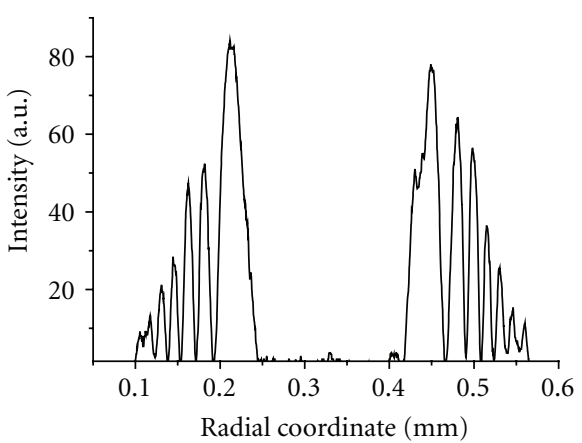

$z=8.3 \mathrm{~mm}$

(b5)

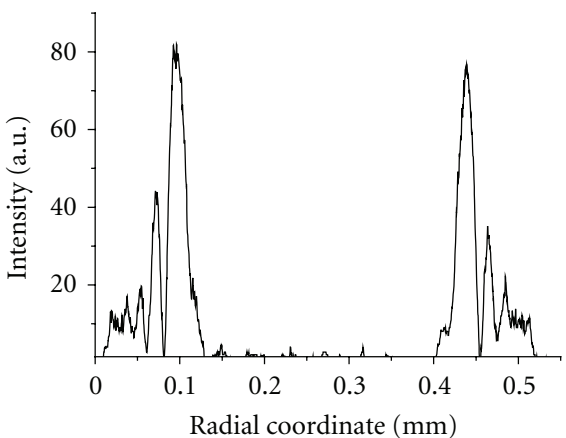

$z=8.8 \mathrm{~mm}$

(b6)

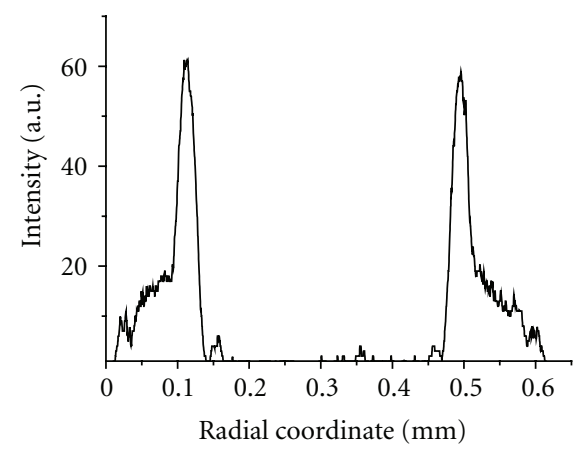

$-z=9.3 \mathrm{~mm}$

(b7)

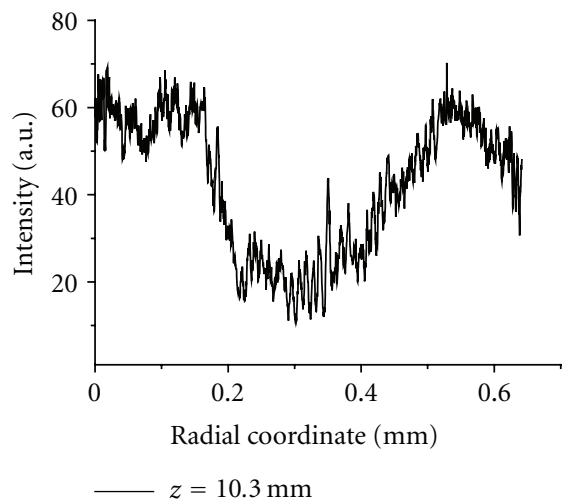

(b8)

(b)

FIGURE 12: ((a1)-(a8)) Experimentally registered transverse structure of the field at different distances after the lens and its one-dimensional cross-sections ((b1)-(b8)). 


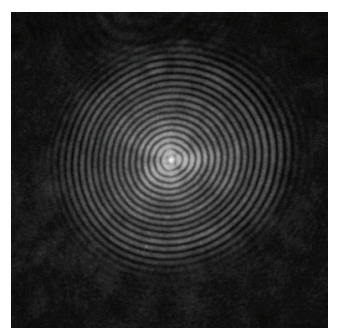

(a)

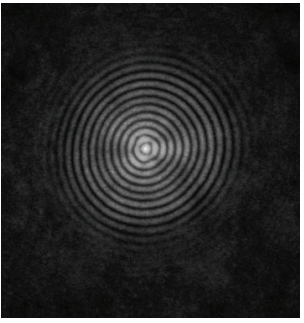

(b)
FIGURE 13: Bessel-like transverse structure of a field at the distance of $z=5.45 \mathrm{~cm} \mathrm{(a)} \mathrm{and} 5.75 \mathrm{~cm} \mathrm{(b).}$

place the composition of two conical beams with different cone angles. To be more pictorial, in right-hand Figure 12(a1) the centers of the beams are shifted relative to each other. This is achieved by shifting the axicon relative to the optical axis. In so doing the Figure 12(a1) corresponds to the case where on the beam axis there takes place the intensity minimum, that is, the destructive interference. And this situation was repeated at a very small period along the $z$-axis. Note that these oscillations of intensity on the beam axis may also be regarded as some version of self-imaging of optical mini-bottle beam (see also [13, 21, 22]).

Figure 12(a3) corresponds to the position of the registration plane in the region of the intensity maximum preceding the bottle beam (see Figure 6). Here, as noted above, a multibeam interference is realized, because the angular dispersion of rays is close to zero. Figures 12(a3)12(a7) show the dynamics of sweeping the bottle beam. Here, on the whole, there is a good agreement with the calculation, except that the periphery zone of the bottle beam is a less extensive one. This seems to be related to neglect of the real structure of the axicon in the vicinity of its vertex where there is the roundness (see $[9,23])$. Figure $12(\mathrm{a} 8)$ refers to the bottom of this bottle beam.

The subsequent change of the field is related to the changeover to beams of Bessel type. Examples of such a beam are given in Figure 13. Here with distance from first lens, the period of intensity oscillations decreases and such a tendency persists up to the far zone [20].

\section{Conclusion}

A study is made of the structure of the light field which is formed by an axicon with a small cone angle and lens with a strong spherical aberration. Basic emphasis is layered on the analysis of the near field after the lens. The calculation of this field is carried out by the methods of geometrical optics and diffraction integral. The results of the calculation as well as experimental measurements have shown that one can single out a few types of the field structure, which are qualitatively different. The first structure is characterized, first of all, by the intensive axial maximum arising as a result of the constructive interference of a great number of conic waves under condition of a small angular dispersion. Also, there is a zone of interference of two conical beams, which forms a characteristic interference pattern with intensity beats. After the mentioned maximum, the bottle beam is formed, the periphery region of which is at first an oscillating one and is practically transformed into one broad ring. At the last state the bottle beam is transformed into the Bessel-type field, its cone angle decreasing with distance up to zero value. This field is of interest by itself and its study in detail is planned to perform later on.

The presence of the spherical aberration also tells on that the near field decreases in size, which would be of practical interest as well. These sizes may be controllable on account of using the spatial degree of freedom of the scheme-distance between the axicon and lens. An additional feasibility of controlling the structure is realized, while changing the values of spherical aberration via changing the diameter of an incident beam as well as the modulation of the refraction index of the lens.

\section{References}

[1] J. H. McLeod, "Axicons and their uses," The Journal of the Optical Society of America, vol. 50, pp. 166-169, 1960.

[2] P. A. Belanger and M. Rioux, "Ring pattern of a lens-axicon doublet illuminated by a gaussian beam," Applied Optics, vol. 17, no. 7, pp. 1080-1086, 1978.

[3] Z. Bouchal, J. Wagner, and M. Olivik, "Bessel beams in the focal region," Optical Engineering, vol. 34, no. 6, pp. 16801688, 1995.

[4] S. De Nicola, "On-axis focal shift effects in focused truncated J0 Bessel beams," Pure and Applied Optics, vol. 5, no. 6, pp. 827-831, 1996.

[5] B. Lü, W. Huang, B. Zhang, F. Kong, and Q. Zhai, "Focusing properties of Bessel beams," Optics Communications, vol. 131, no. 4-6, pp. 223-228, 1996.

[6] C. Parigger, Y. Tang, D. H. Plemmons, and J. W. L. Lewis, "Spherical aberration effects in lens-axicon doublets: theoretical study," Applied Optics, vol. 36, no. 31, pp. 8214-8221, 1997.

[7] K. Ait-Ameur and F. Sanchez, "Gaussian beam conversion using an axicon," Journal of Modern Optics, vol. 46, no. 10, pp. 1537-1548, 1999.

[8] S. Chávez-Cerda and G. H. C. New, "Evolution of focused Hankel waves and Bessel beams," Optics Communications, vol. 181, no. 4, pp. 369-377, 2000.

[9] B. Dépret, P. Verkerk, and D. Hennequin, "Characterization and modelling of the hollow beam produced by a real conical lens," Optics Communications, vol. 211, no. 1-6, pp. 31-38, 2002.

[10] J. C. Gutiérrez-Vega, R. Rodríguez-Masegosa, and S. ChávezCerda, "Focusing evolution of generalized propagation invariant optical fields," Journal of Optics A, vol. 5, no. 3, pp. 276282, 2003.

[11] M. De Angelis, L. Cacciapuoti, G. Pierattini, and G. M. Tino, "Axially symmetric hollow beams using refractive conical lenses," Optics and Lasers in Engineering, vol. 39, no. 3, pp. 283-291, 2003.

[12] M. D. Wei, W. L. Shiao, and Y. T. Lin, "Adjustable generation of bottle and hollow beams using an axicon," Optics Communications, vol. 248, no. 1-3, pp. 7-14, 2005.

[13] B. P. S. Ahluwalia, W. C. Cheong, X. C. Yuan et al., "Design and fabrication of a double-axicon for generation of tailorable self-imaged three-dimensional intensity voids," Optics Letters, vol. 31, no. 7, pp. 987-989, 2006. 
[14] M. D. Wei, "Generation of bottle beam by focusing a super-Gaussian beam using a lens and an axicon," Optics Communications, vol. 277, no. 1, pp. 19-23, 2007.

[15] Z. Liu, J. Dai, X. Zhao, X. Sun, S. Liu, and M. A. Ahmad, "Generation of dark hollow beam by use of phase-only filtering," Optics and Lasers in Engineering, vol. 47, no. 11, pp. 1250-1253, 2009.

[16] J. H. Lin, M. D. Wei, H. H. Liang, K. H. Lin, and W. F. Hsieh, "Generation of supercontinuum bottle beam using an axicon," Optics Express, vol. 15, no. 6, pp. 2940-2946, 2007.

[17] J. Arlt and M. J. Padgett, "Generation of a beam with a dark focus surrounded by regions of higher intensity: the optical bottle beam," Optics Letters, vol. 25, no. 4, pp. 191-193, 2000.

[18] I. Manek, Y. B. Ovchinnikov, and R. Grimm, "Generation of a hollow laser beam for atom trapping using an axicon," Optics Communications, vol. 147, no. 1-3, pp. 67-70, 1998.

[19] E. M. Wright, J. Arlt, K. Dholakia, and J. Soneson, "Optical dipole traps and atomic waveguides based on Bessel light beams," Physical Review A, vol. 63, no. 6, Article ID 063602, 8 pages, 2001.

[20] V. Belyi, A. Forbes, N. Kazak, N. Khilo, and P. Ropot, "Bessellike beams with z-dependent cone angles," Optics Express, vol. 18, no. 3, pp. 1966-1973, 2010.

[21] B. P. S. Ahluwalia, X. C. Yuan, and S. H. Tao, "Generation of self-imaged optical bottle beams," Optics Communications, vol. 238, no. 1-3, pp. 177-184, 2004.

[22] R. Vasilyeu, A. Dudley, N. Khilo, and A. Forbes, "Generating superpositions of higher-order Bessel beams," Optics Express, vol. 17, no. 26, pp. 23389-23395, 2009.

[23] S. Akturk, B. Zhou, B. Pasquiou, M. Franco, and A. Mysyrowicz, "Intensity distribution around the focal regions of real axicons," Optics Communications, vol. 281, no. 17, pp. 42404244, 2008. 

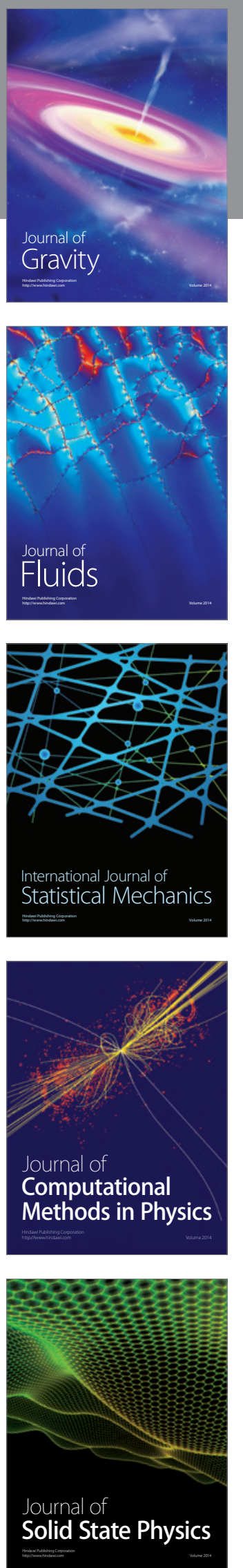

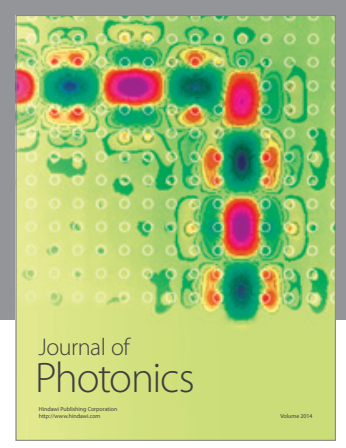

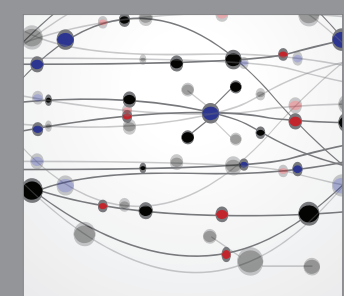

The Scientific World Journal
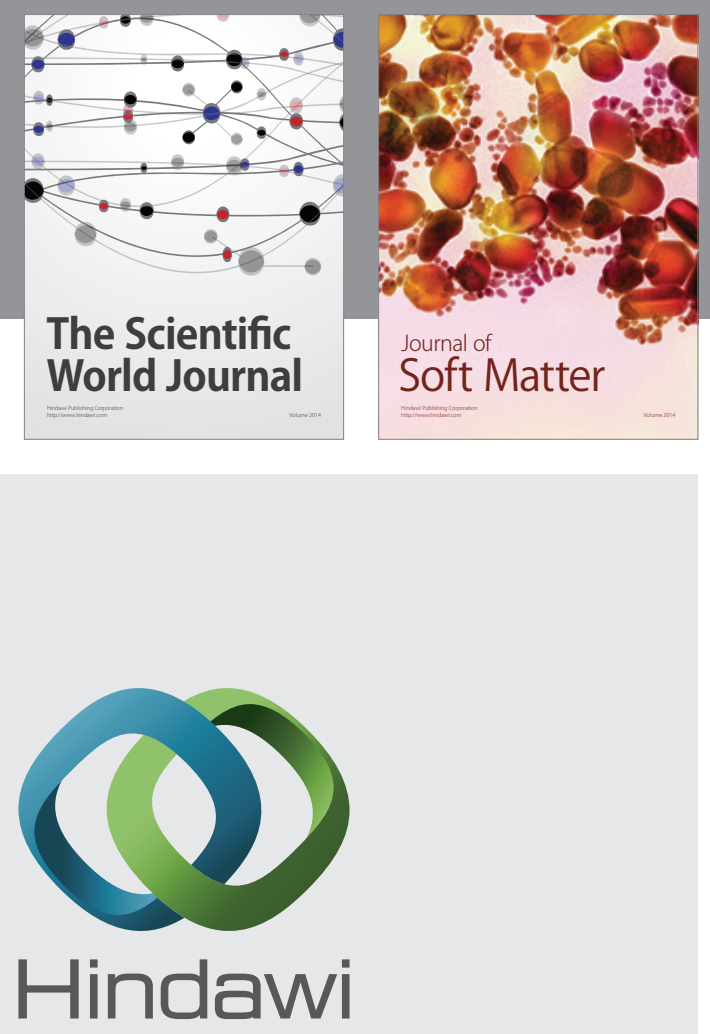

Submit your manuscripts at

http://www.hindawi.com
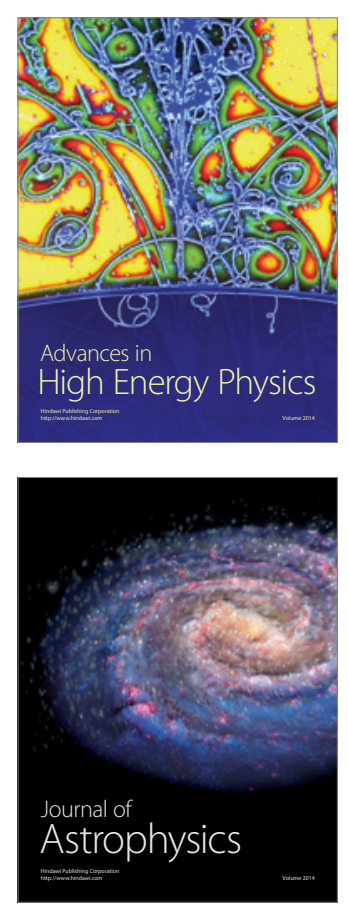
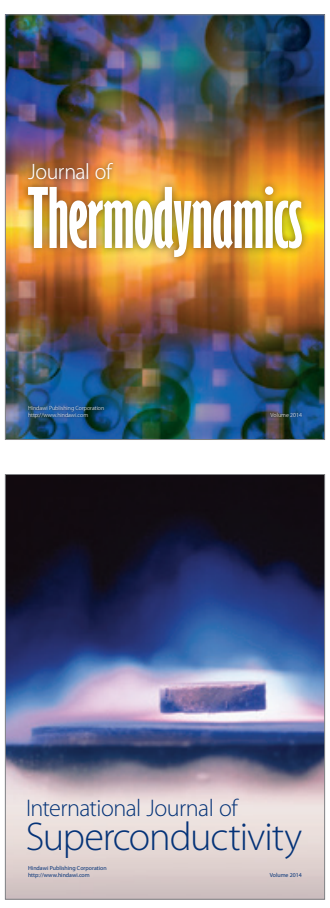
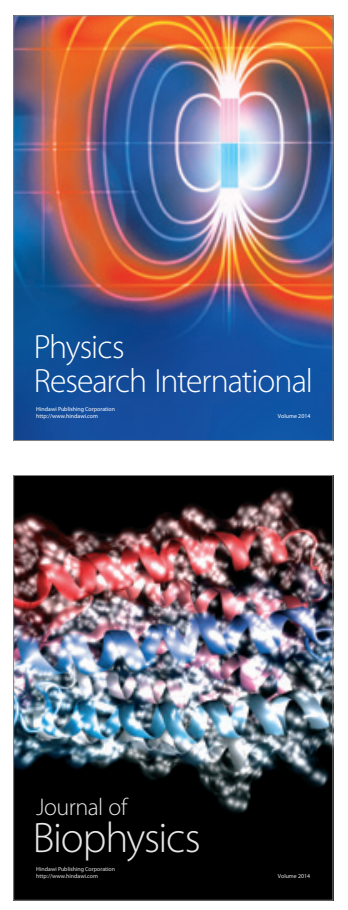
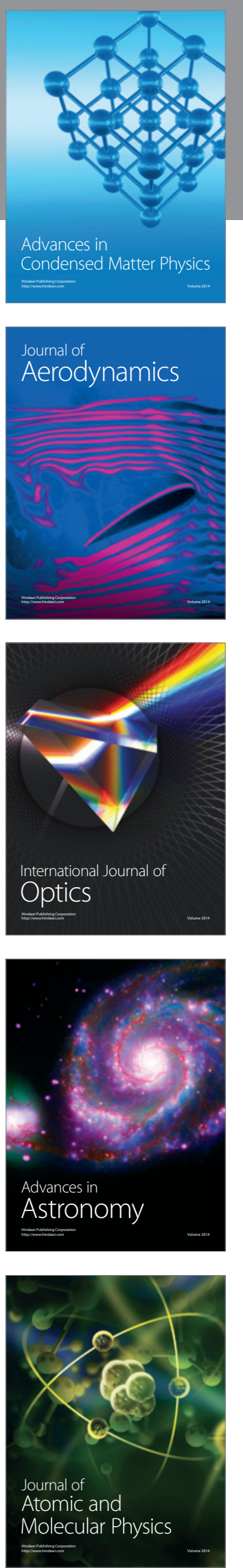